\title{
LIS1 Association With Dynactin is Required for Nuclear Motility and Genomic Union in the Fertilized Mammalian Oocyte
}

\author{
Christopher Payne, ${ }^{1,2}$ Justin C. St. John, ${ }^{3}$ João Ramalho-Santos, ${ }^{2,4}$ and \\ Gerald Schatten ${ }^{2 *}$ \\ ${ }^{1}$ Program in Molecular and Cellular Biosciences, Department of Cell and \\ Developmental Biology, Oregon Health and Science University, Portland \\ ${ }^{2}$ Pittsburgh Development Center, Magee-Womens Research Institute, Departments \\ of Obstetrics, Gynecology and Reproductive Sciences, and Cell Biology and \\ Physiology, University of Pittsburgh School of Medicine Pittsburgh, Pennsylvania \\ ${ }^{3}$ Reproductive Biology and Genetics Group, Department of Medicine, University of \\ Birmingham, Birmingham, United Kingdom \\ ${ }^{4}$ Center for Neuroscience and Cell Biology, Department of Zoology, University of \\ Coimbra, Coimbra, Portugal
}

\begin{abstract}
Mutations in the human LIS1 gene cause the devastating brain disorder lissencephaly. LIS1 also regulates microtubule dynamics; it interacts with the molecular motor cytoplasmic dynein and its cofactor dynactin, and is necessary for neuronal migration. Recently, LIS1 has been suggested to mediate pronuclear migration during fertilization. Here we use rhesus monkey and bovine oocytes, as well as pronucleate-stage bovine zygotes, to determine: Lis1 RNA expression using reverse transcriptionpolymerase chain reaction; LIS1 protein association with dynactin using immunoprecipitation, Western blot analysis, and immunocytochemistry; and LIS1 function in mediating genomic union using antibody transfection. We find that Lis1 RNA expression increases during fertilization, that LIS1 and dynactin subunit p150/Glued co-immunoprecipitate and co-localize to pronuclear surfaces, and that anti-LIS1 antibodies transfected into zygotes dramatically inhibit pronuclear migration and apposition. LIS1 is, therefore, essential to mediate genomic union in a process that involves the dynein-dynactin complex. These results shed light on an additional role for LIS1 and raise implications for human reproduction. Cell Motil. Cytoskeleton 56: 245-251, 2003. () 2003 Wiley-Liss, Inc.
\end{abstract}

Key words: nuclear movement; microtubules, molecular motor; fertilization; LIS1 inhibition

\section{INTRODUCTION}

Human type I lissencephaly is a severe brain disorder caused by the failure of neurons to migrate from the paraventricular zone to the cerebral cortex during development [Dobyns et al., 1993]. This results in disorganized cortical layers and reduced gyri, inducing epilepsy and severe mental retardation with death at an early age. Haplo-insufficiency of the Lis1 gene product leads to both isolated lissencephaly sequence (ILS) and Miller-Dieker Syndrome (MDS), which together comprise a majority of cases seen in the clinic [Lo Nigro et al., 1997]. Whereas total loss of Lis 1 is embryonic lethal, heterozygous mutations generated in mice result in neuronal migration defects
[Hirotsune et al., 1998]. The LIS1 protein, also a subunit of platelet-activating factor acetylhydrolase

Contract grant sponsor: Fundação para a Ciência e Tecnologia (FCT), Portugal; Contract grant number: POCTI/ESP/38049/2001; Contract grant sponsor: NIH.

*Correspondence to: Gerald Schatten, Pittsburgh Development Center, Magee-Womens Research Institute, Departments of Obstetrics, Gynecology and Reproductive Sciences, and Cell Biology and Physiology, University of Pittsburgh School of Medicine, Pittsburgh, PA 15213.

E-mail: gschatten@magee.edu

Received 23 April 2003; accepted 10 September 
(PAFAH), has been shown to modulate microtubule dynamics in vitro [Sapir et al., 1997].

LIS1 shares $42 \%$ identity with NUDF, one of many nuclear distribution (NUD) proteins in the filamentous fungus Aspergillus nidulans [Xiang et al., 1995]. During development in $A$. nidulans, nuclei migrate into the germtube and distribute within the cell, and many of the genes that regulate this process share striking homology to members of the cytoplasmic dynein-dynactin motor complex, including $N U D M$, a homologue of dynactin subunit $p 150^{\text {Glued }}$ [Xiang and Morris, 1999]. Mutations in Drosophila LIS1, C. elegans LIS1, and the yeast homologue, $P A C 1$, generate dynein-related defects in nuclear migration, nuclear orientation, and oogenesis [Swan et al., 1999; Dawe et al., 2001; Geiser et al., 1997].

Fertilization in most mammals requires a sperm aster, a radial array of microtubules nucleated by the sperm centrosome, upon which the egg-derived female pronucleus migrates to the sperm-derived male pronucleus in a process mediated by the dynein-dynactin motor complex [Payne et al., 2003; Reinsch and Karsenti, 1997; Schatten, 1994]. Surprisingly, rodents do not utilize a sperm aster during fertilization, precluding their use for investigating sperm aster-mediated motility in mammals [Schatten, 1994], though Lis1 expression was recently identified in mouse oocytes and zygotes [Cahana and Reiner, 1999]. Because of the relationship that exists among LIS1, dynein, dynactin, and nuclear migration in non-mammalian species, we questioned whether Lis1 is expressed in rhesus monkey and bovine oocytes, and whether LIS1 is required for pronuclear motility and union in bovine zygotes.

\section{MATERIALS AND METHODS}

\section{In Vitro Maturation and In Vitro Fertilization}

Rhesus monkey and bovine in vitro maturation, along with bovine in vitro fertilization, were carried out according to standard protocols [Hewitson et al., 1998; Navara et al., 1994]. Rhesus oocytes were obtained from the Assisted Reproductive Technology (ART) core facilities at the Oregon National Primate Research Center and Pittsburgh Development Center, bovine oocytes were obtained from a local abattoir and BOMED, Inc. (Madison, WI), and bull semen was obtained from American Breeders Service (DeForest, WI). Rhesus oocytes were cultured at $37^{\circ} \mathrm{C}$ under $5 \% \mathrm{CO}_{2}$, and bovine oocytes and sperm were incubated at $39^{\circ} \mathrm{C}$ under $5 \% \mathrm{CO}_{2}$, for up to $20 \mathrm{~h}$.

\section{RNA Isolation and Reverse Transcription- Polymerase Chain Reaction}

Total RNA from rhesus and bovine oocytes, as well as from bovine zygotes, was isolated and prepared using the
StrataPrep ${ }^{\circledR}$ Total RNA Microprep Kit following the manufacturer's protocol (Stratagene, La Jolla, CA). Reverse transcription (RT) and polymerase chain reaction (PCR) amplification of cDNA were performed on the PTC-200 DNA Engine Cycler from MJ Research, using the ProSTAR $^{\text {TM }}$ HF Single-Tube RT-PCR System (High Fidelity) from Stratagene. Oligonucleotide primers designed for Lis1 RT-PCR yield a 329-bp product: 5'-GTCGTAGCAACAAAGGAATGC-3' and 5'-CGCTTGTTCTTGTAATCCCATAC-3' (primers LIS1(71)1001F and LIS1(71)1329R, respectively) [Lo Nigro et al., 1997]. For $\beta$-actin RT-PCR, primers yield a 225-bp product: 5'-CTGGCATTGTCATGGACTCT-3' and 5'-TCGAAGTCTAGGGCGACATA$3^{\prime}$. Control primers provided by the ProSTAR ${ }^{\mathrm{TM}}$ system were used to amplify control products with a size of $500 \mathrm{bp}$. This control amplification was recommended by the manufacturer to ensure that the kit reagents were working properly. The reverse transcription and PCR steps occurred consecutively in the same tube: reverse transcription was performed at $42^{\circ} \mathrm{C}$ for $15 \mathrm{~min}$ and PCR amplification was carried out with an initial denaturation at $95^{\circ} \mathrm{C}$ for $1 \mathrm{~min}$, followed by 40 cycles of denaturation at $95^{\circ} \mathrm{C}$ for $30 \mathrm{~s}$, annealing at $55^{\circ} \mathrm{C}$ for $30 \mathrm{~s}$, and extension at $68^{\circ} \mathrm{C}$ for $1 \mathrm{~min}$. A final extension of the PCR products occurred at $68^{\circ} \mathrm{C}$ for $10 \mathrm{~min}$. A negative control reaction was carried out excluding the reverse transcriptase from the reaction mixture. RT-PCR fragments were electrophoresed on $2.5 \%(\mathrm{w} / \mathrm{v})$ agarose gels and referenced with the 100-bp ladder by Life Technologies. Visualization and analysis of RT-PCR products was achieved using the Gel Doc 2000 ${ }^{\mathrm{TM}}$ system from Bio-Rad (Richmond, CA). Quantitation of RT-PCR was performed by using the software included with the Gel Doc Imaging system, comparing levels of the Lis1 products with the normalized standards of the $\beta$-actin products.

\section{DNA Sequencing and Sequence Alignment}

PCR products were end-sequenced using the Lis1 oligonucleotide primers with dye terminators (373 DNA ABI Sequencer; Perkin-Elmer Biosystems). The cDNA sequences for the rhesus and two bovine Lis1 fragments were submitted to GenBank and given the accession numbers AY260741, AY260742, and AY260743, respectively. Sequence alignments were performed using ClustalW Multiple Sequence Alignment computer program, comparing the bovine and rhesus cDNA sequences to human LIS1 exons 8, 9, and 10 (GenBank accession numbers U72339, U72340, and U72341, respectively) [Lo Nigro et al., 1997]. Human LIS1 coding sequence used in the alignment encompasses bases 534-650 from U72339, bases 295-396 from U72340, and bases 404513 from U72341. Deduced protein sequence was generated from the bovine and rhesus nucleotide sequences. 


\section{Immunoprecipitation and Western Blot Analysis}

SDS-PAGE and Western Blotting of bovine oocytes and zygotes were performed following standard protocols [Harlow and Lane, 1988], and immunoprecipitations of $\mathrm{p} 150^{\text {Glued }}$ dynactin were carried out using Protein A Sepharose 4 Fast Flow medium according to the manufacturer's recommendations (AP Biotech). A concentration of $2.5 \mu \mathrm{g} / \mathrm{ml}$ anti-p15 ${ }^{\text {Glued }}$ antibody was used for the immunoprecipitation reactions. Mature bovine oocytes were either lysed immediately or fertilized in vitro and cultured until $16 \mathrm{~h}$ post-insemination, at which time they were lysed and prepared for antibody incubations. Protein concentrations were determined by the Bradford assay, and equal amounts of protein were added to each lane of polyacrylamide gels. Samples were loaded onto $4-20 \%$ gradient Tris- $\mathrm{HCl}$ gels, and processed by SDS-PAGE and Western Blotting onto PVDF membranes. Gels were silver stained to verify the presence and relative amounts of protein. LIS1 was identified on the Western blots using goat and rabbit polyclonal antibodies (Santa Cruz Biotechnology, Santa Cruz, CA) at a concentration of $1.25 \mu \mathrm{g} / \mathrm{ml}$. Monoclonal anti-dynactin p15 $5^{\text {Glued }}$ antibody (BD Biosciences) was also used at $1.25 \mu \mathrm{g} / \mathrm{ml}$. HRP-conjugated secondary antibodies were obtained from Molecular Probes (Eugene, OR) and Jackson ImmunoResearch (West Grove, PA) and used at a 1:5,000 dilution. Protein bands were visualized using the ECL Plus system and HyperFilm (AP Biotech), referenced to Kaleidoscope pre-stained standards (BioRad), and analyzed by a densitometer.

\section{Immunocytochemistry and Antibody Transfection}

All zygotes were processed for immunocytochemistry (ICC) using previously described methods [Navara et al., 1994; Payne and Schatten, 2003], with fixation in $2 \%$ formaldehyde for $40 \mathrm{~min}$ at room temperature, followed by permeabilization with $0.1 \% \mathrm{TX}-100$ in $10 \mathrm{mM}$ PBS for an additional $40 \mathrm{~min}$. After blocking with $0.3 \%$ BSA in $10 \mathrm{mM}$ PBS, samples were incubated with primary antibodies. LIS1 was identified by ICC using goat and rabbit polyclonal antibodies (Santa Cruz Biotechnology) at a concentration of $12.5 \mu \mathrm{g} / \mathrm{ml}$. Monoclonal antidynactin $\mathrm{p} 150^{\mathrm{Glued}}$ antibody (BD Biosciences) was also used at $12.5 \mu \mathrm{g} / \mathrm{ml}$. Control experiments were performed by pre-absorbing the anti-LIS1 antibodies with LIS1 blocking peptide (Santa Cruz Biotechnology), by preincubating the anti-dynactin $\mathrm{p} 150^{\text {Glued }}$ antibodies with human endothelial cell lysates provided by the Pittsburgh Development Center, and by using pre-immune mouse IgG antibodies (Chemicon, Temecula, CA) at $12.5 \mu \mathrm{g} /$ $\mathrm{ml}$. Following washes, secondary antibodies were then applied, using AlexaFluor-conjugated antibodies (Molecular Probes) at a 1:200 dilution. Samples were then stained with $10 \mu \mathrm{g} / \mathrm{ml}$ TOTO-3 (Molecular Probes) to label the DNA. Fixed zygotes were imaged using a Leica TCS SP2 spectral confocal microscope at laser lines 488, 568 , and $633 \mathrm{~nm}$.

Antibody transfection of bovine zygotes at $12 \mathrm{~h}$ post-insemination was achieved using the Chariot ${ }^{\mathrm{TM}}$ reagent following the manufacturer's protocol (Active Motif). Briefly, for each antibody transfection into $20 \mathrm{zy}-$ gotes, a 1:5 dilution of anti-LIS1 antibodies, or a 1:20 dilution of pre-immune IgG antibodies, was mixed with a 1:10 dilution of Chariot ${ }^{\mathrm{TM}}$ reagent in a total volume of $20 \mu \mathrm{l}$. This mixture was then incubated at room temperature for $30 \mathrm{~min}$. Following this antibody-Chariot ${ }^{\mathrm{TM}}$ binding step, the entire $20 \mu \mathrm{l}$ volume was added to one well of a 96-well plate containing 20 zygotes in $80 \mu \mathrm{l}$ of protein-free TL-Hepes medium. The samples were incubated at $39^{\circ} \mathrm{C}$ for $1 \mathrm{~h}$, after which an additional $100 \mu \mathrm{l}$ of protein-containing TL-Hepes medium was added to the well; samples were cultured for an additional $7 \mathrm{~h}$, and fixed for immunocytochemistry at $20 \mathrm{~h}$ post-insemination. For these transfection experiments, antibodies were dialyzed overnight using Slide-A-Lyzer cassettes (Pierce, Rockford, IL) in multiple changes of $10 \mathrm{mM}$ PBS to remove sodium azide from the storage buffer.

\section{RESULTS \\ Cloning and Expression of Lis1 in Rhesus Monkey and Bovine Oocytes and Zygotes}

To characterize the expression of Lis1 in mammalian oocytes that utilize a sperm aster during fertilization, we isolated total RNA from rhesus monkey and bovine oocytes and performed RT-PCR. Amplification of cDNA was performed using oligonucleotide primers that recognize a coding region within the human LISI gene and that were previously used to predict outcome associated with ILS and MDS [Lo Nigro et al., 1997]. This portion of the gene is relevant to the current study, as it is a region that might show genetic variability across species. RT-PCR using these primers yielded 329-bp products (Fig. 1A). Two bovine Lis1 products (1031A and 1031B) and one rhesus Lis1 product $(\mathrm{Rh} 6)$ were then sequenced and compared to the human LIS1 coding sequence of that region (Fig. 1B). Human Lis1 is 95\% identical to rhesus Lis1 and 91\% identical to bovine Lis1 over the length of the isolated cDNA. This high degree of homology is not entirely surprising, since the recent comparison between human and rhesus UDP-glucuronosyl-transferase also revealed $95 \%$ identity, reflecting the extensive conservation of sequence among primates [Dean et al., 2002]. The deduced protein sequence is shown in Figure 1C.

Mammalian Lis1 expression at fertilization was examined further by performing RT-PCR and comparing 
A

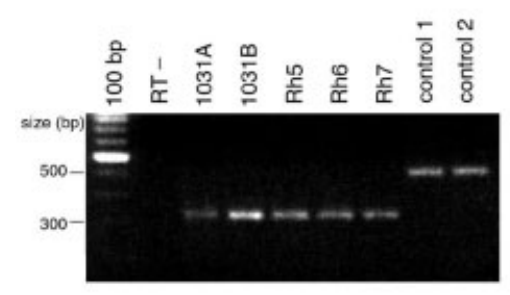

C

HEHVVECISWAPESSYSSISEATGSETK KSGKPGPFLLSGSRDKTIKMWDVSTG MCLMTLVGHDNWVRGVLFHSGGKFIL SCADDKTLRVWDYKNKR
B

1031A ATCACTTGGCCTTGATCTT CGACAMCACGAGCATGTGGTAGATC

1031B GTTTCTTCGCCTTGATCTTCGACANCATGAGCATGTGGTAGAATG

Rh6 GGAACTAGAGCTTGATCTCCGAGAGCATGAGCATGTGGTAGAATG

Human GGATCCAAGGCTGACCTCCGACACCATGAGCATGTCGTAGATC
1031 A CATTTCCTGGGCTCCTGMUGCTCATATTCT TCCATCTCTGAAGCAACAGGATCTCAGAC
1031 B CATTTCCTGGGCTCCTGANAGCTCATATTCTTCCATCTCTGAAGCAACAGGATCTCAGAC 1031 B CATTTCCTGGGCTCCTGAAAGCTCATATTCTTCCATCTCTGAAGCAACAGGATCTCAGAC Rh6 CATTTCCTGGGCTCCAGAAAGCTCATATTCCTCCATCTCTGAAGCAACAGGATCTGAGAC CATTTCCTGGGCTCCAGAAAGTCATATTCCTCCATCTCTGAAGCALCAGGATCTGAGAC

1031 A TANWAAGTGGCANACCTGGGCCATTCTTACTGTCCGGATCCAGGGACAAGACTATCAA
10318 TAAAAAAAGTGGCANACCTGGGCCATTCTTACTGTCCGGATCCAGGGACAAGACTATCAA 10318 TAAAAAAAGTGGCAAACCTGGGCCATTCTTACTGTCCGGATCCAGGGACAAGACTATCAA
Rh6 TAAAAAAAGTGTAAACCTGGGCCATTCTTGCTGTCTGGATCCAGAGACAAGACTATTAA HUMAO TAAAAAAAGTGGTAAACCTGGGCCATTCTIGCTGTCTGGATCCAGAGACAAGACTATTAA

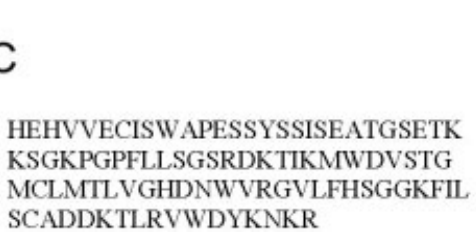

Fig. 1. RT-PCR and cDNA sequence analysis of bovine and rhesus monkey Lis1. A: Detection of the 329-bp Lis1 product, reverse transcribed and amplified from total RNA, isolated from bovine (1031A, 1031B) and rhesus (Rh5, Rh6, and Rh7) oocytes. Positive controls 1 and 2 yield a 500-bp product, and negative control (RT-) shows no product. B: Sequence comparison among bovine (1031A, 1031B),

the levels of Lis1 products isolated from unfertilized bovine oocytes and zygotes against the levels of $\beta$-actin products (Fig. 2A). Semi-quantitation of the Lis1 products indicates that levels in the zygotes are nearly double those in the oocytes $(\sim 190 \%)$. These results suggest that Lis1 mRNA might be transcribed in the fertilized zygote. Recent evidence has shown that 1-cell bovine embryos are indeed transcriptionally and translationally active for genes important for embryonic development [Memili and First, 1999]. Lis1 transcription has also been reported to occur in mouse zygotes, indicating the importance of this gene's expression in the early mammalian embryo [Cahana and Reiner, 1999].

\section{LIS1 Associates With Dynactin in Pronucleate- Stage Bovine Zygotes}

Western blot analysis was performed to determine whether LIS1 translation occurs during fertilization. LIS1 protein is detected here as a single $45-\mathrm{kD}$ band in unfertilized bovine oocytes and zygotes (Fig. 2B), with densitometry analysis showing levels approximately onethird $(\sim 30 \%)$ higher in zygotes than in oocytes. This putative increase in mRNA and protein expression in the zygote suggests a potential function for LIS1 during fertilization and early embryogenesis.

LIS1 interacts with the $150^{\text {Glued }}$ subunit of dynactin in embryonic, neonatal, and adult mammalian neurons, and its association with dynactin is particularly enriched at the cortical plate and marginal zone of the embryonic brain, rhesus (Rh6), and human Lis1 cDNA. Data are taken from GenBank as listed in Materials and Methods, and sequence analysis was performed with the Multiple Sequence Alignment computer program using the ClustalW algorithm. Conserved residues are highlighted with gray boxes. C: The deduced protein sequence of this portion of the Lis1 gene, generated from the bovine and rhesus nucleotide sequences.
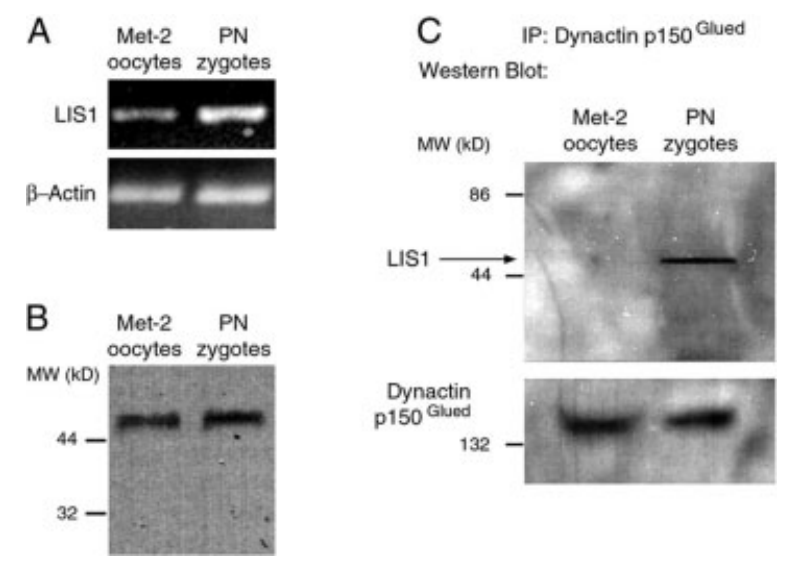

Fig. 2. LIS1 RNA expression, protein expression and co-immunoprecipitation with dynactin. A: RNA from 5 meiotically arrested (Met-2) bovine oocytes or 5 pronucleate $(\mathrm{PN})$ bovine zygotes was isolated, reverse transcribed, and amplified for each RT-PCR reaction. Lis 1 and control $\beta$-actin products were electrophoresed, imaged, and quantified. Semi-quantitative comparison between the levels of Lis1 products in oocytes and zygotes and the normalized standards of $\beta$-actin products suggests that Lis1 transcription may occur upon fertilization. B: Protein from bovine Met-2 oocytes and PN zygotes was extracted, resolved by SDS-PAGE, and subjected to Western blot analysis using anti-LIS1 antibodies. Single 45-kD bands are detected in both oocytes and zygotes. C: SDS-PAGE and Western blot analysis following immunoprecipitation (IP) of dynactin $\mathrm{p} 150^{\text {Glued }}$ from bovine Met-2 oocytes and PN zygotes. LIS1 is enriched in the dynactin immunoprecipitate prepared from zygotes, but not from oocytes. Dynactin $150^{\text {Glued }}$ is detected in both samples. 

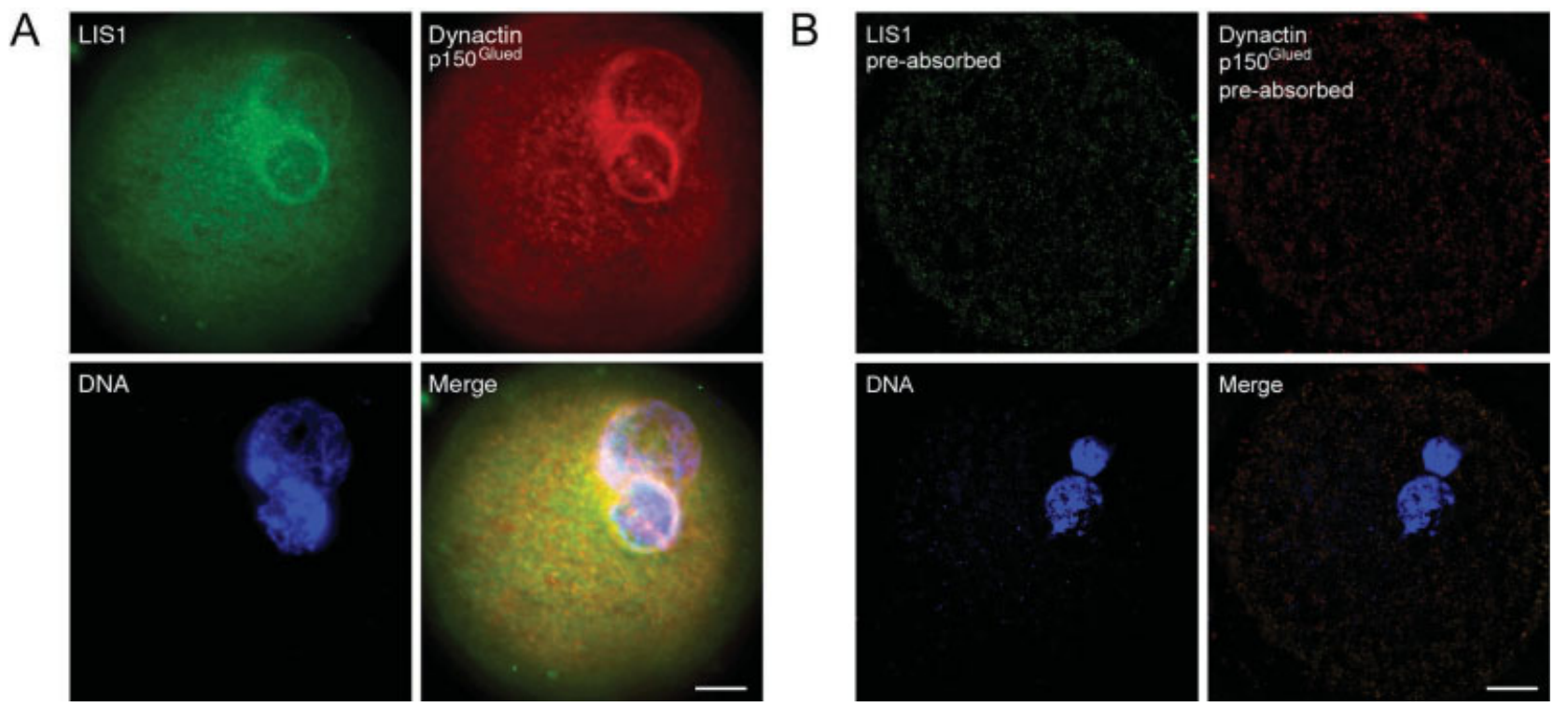

Fig. 3. LIS1 and dynactin co-localize around the pronuclear surfaces in zygotes. A: Pronucleate-stage bovine zygote shows both LIS1 (green) and Dynactin p150 Glued (red) concentrated around the two pronuclei (DNA; blue). Co-localization is detected in the three-channel overlay (Merge). B: Preabsorption of anti-LIS1 antibodies with their antigens and pre-incubation of anti-p $150^{\text {Glued }}$ antibodies with human endothelial cell lysates result in a loss of LIS1 and dynactin detection in zygotes. Scale bar $=10$ $\mu \mathrm{m}$.

sites of neuronal migration [Smith et al., 2000]. We, therefore, questioned whether LIS1 associates with dynactin in bovine zygotes during pronuclear migration. Co-immunoprecipitation experiments show that the anti-dynactin p150 Glued antibody pulls down LIS1 from pronucleate-stage zygotes, but not from unfertilized oocytes (Fig. 2C). The p150 ${ }^{\text {Glued }}$ subunit is enriched in both samples. This result indicates that LIS1 interacts with dynactin at fertilization, perhaps to mediate pronuclear motility.

\section{Co-Distribution of LIS1 and Dynactin to Pronuclear Surfaces}

The distribution of LIS1 and dynactin $\mathrm{p} 150^{\text {Glued }}$ during pronuclear migration and apposition was then characterized by confocal microscopy. Both proteins localize at the surfaces of the two pronuclei (Fig. 3A). While some dim cytoplasmic foci can be seen for LIS1 and dynactin, both proteins concentrate along the pronuclear rims. Pre-absorption of anti-LIS1 antibodies with their antigens and pre-

Fig. 4. LIS1 is required for pronuclear apposition. Left: Normal inter-pronuclear distances are observed when control IgG antibodies are transfected into bovine zygotes. LIS1 concentrates around the two pronuclei, with additional punctate cytoplasmic foci. Right: Transfection using anti-LIS1 antibodies inhibits pronuclear apposition, while the distribution of LIS1 resembles control conditions. Scale bar $=10$ $\mu \mathrm{m}$.

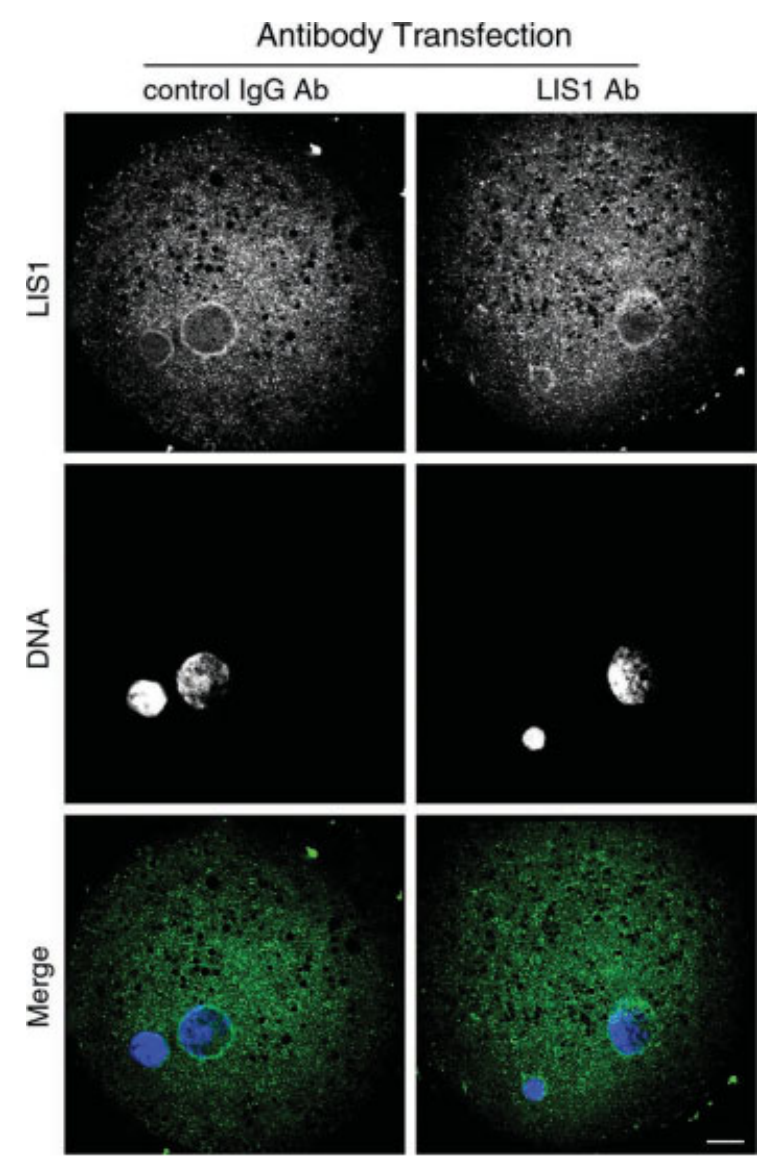


TABLE I. Anti-LIS1 Antibodies Inhibit Pronuclear Union During Fertilization

\begin{tabular}{lcr}
\hline Transfection of zygotes at & \multicolumn{2}{c}{ Distance between pronuclei at $20 \mathrm{~h}$} \\
\cline { 2 - 3 } $12 \mathrm{~h}$ with Chariot & $\geq 10 \mu \mathrm{m}$ apart & $<10 \mu \mathrm{m}$ apart \\
reagent + antibodies: & $0 \%(0 / 92)$ & $100 \%(92 / 92)$ \\
Pre-immune mouse $\mathrm{IgG}$ & $81 \%(69 / 85)$ & $19 \%(16 / 85)$ \\
\hline Anti-LIS1 &
\end{tabular}

incubation of anti-p $150^{\text {Glued }}$ antibodies with human endothelial cell lysates result in no LIS1 or dynactin staining within the zygotes (Fig. 3B). LIS1 has recently been identified to localize at nuclear envelopes in prophase somatic cells [Coquelle et al., 2002], and dynactin has been proposed to interact with cytoplasmic dynein to facilitate nuclear envelope breakdown [Salina et al., 2002]. Another possible function of LIS1 and the dynein-dynactin complex might be to facilitate pronuclear migration.

\section{Requirement of LIS1 for Genomic Union}

Co-localization of LIS1 and dynactin to pronuclear surfaces suggests their importance in mediating genomic union. To determine whether LIS1 is required for pronuclear migration and apposition, we transfected antibodies against LIS1 into pronucleate-stage bovine zygotes using Chariot ${ }^{\mathrm{TM}}$ reagent [Morris et al., 2001]. Antibody transfection occurred after the formation but before the union of the pronuclei, specifically targeting pronuclear motility. Development of the transfected zygotes was allowed until just prior to mitosis, whereupon distances between the two pronuclei were measured to score the inhibition of genomic union. Measured from surface-to-surface, inter-pronuclear distances $\geq 10 \mu \mathrm{m}$ reflect inhibition, with $10 \mu \mathrm{m}$ representing the average pronuclear diameter.

The majority of zygotes transfected with anti-LIS1 antibodies show pronuclei $\geq 10 \mu \mathrm{m}$ apart $(81 \%$; Table I). LIS1 concentrates around both pronuclei and distributes throughout the cytoplasm as punctate foci (Fig. 4). All of the zygotes transfected with pre-immune mouse IgG antibodies display inter-pronuclear distances $<10 \mu \mathrm{m}$ and normal LIS1 distribution (Table I, Fig. 4). We conclude from these data that LIS1 is essential for pronuclear migration and genomic union. Recent observations of $C$. elegans zygotes noted that pronuclear apposition does not occur when the animals are subjected to Lis1 RNAi [Dawe et al., 2001]. Our results here demonstrate a role for LIS1 on nuclear motility during mammalian fertilization, with implications for human infertility.

\section{DISCUSSION}

LIS1 is required for early mouse embryogenesis, with homozygous null mutants exhibiting post-implanta- tion lethality [Hirotsune et al., 1998]. Morphological analysis of homozygous null blastocysts revealed defects in inner cell mass growth and development, resulting in embryonic death prior to neuronal differentiation or migration. While the precise cause of such defects has not yet been identified, the ability of these embryos to develop until implantation suggests that maternally-inherited Lis1 transcripts and protein levels are sufficient in the zygote to permit development in the absence of gene expression. This current study shows that when LIS1 protein is inhibited by antibody transfection into the zygote, specific defects in genomic union are observed.

Co-immunoprecipitation and co-localization of LIS1 with dynactin in the zygote identify a possible role for LIS1 in modulating the dynein-dynactin complex. Recent studies report that LIS1, dynein, and dynactin localize to mitotic kinetochores and microtubule "plus" ends in somatic cells [Faulkner et al., 2000; Coquelle et al., 2002], and that LIS1 interacts with specific regions in the dynein and dynactin molecules [Tai et al., 2002]. Indeed, in unfertilized oocytes LIS1 localizes to regions along the meiotic spindle that are enriched with dynein and dynactin (C. Payne, unpublished observations). Given the proposed role of dynein and dynactin in mediating pronuclear migration and apposition during fertilization [Payne et al., 2003; Reinsch and Karsenti, 1997; Schatten, 1994], LIS1 likely regulates the motor complex at the surfaces of the pronuclei to facilitate nuclear motility and union. Recent evidence shows that when anti-dynein and anti-dynactin antibodies are transfected into pronucleate-stage zygotes, the migration of the female pronucleus is dramatically inhibited [Payne et al., 2003]. Thus, the similarity in outcome of blocking dynein, dynactin, and LIS1 during fertilization suggests their involvement in an interactive complex that mediates pronuclear movement.

It is remarkable that a gene highly expressed in neurons and critical for neuronal migration should also be expressed and functionally important at fertilization. Recent studies illustrate, however, that neurons and gametes share more commonalities than were once appreciated. The RNA binding protein CPEB (cytoplasmic polyadenylation element binding protein), for example, regulates key functions in both cell types [Richter, 2001], and SPNR (spermatid perinuclear RNA-binding protein) shows abundant expression in brain, ovary, and testis [Pires-daSilva et al., 2001]. Thus, proteins essential for proper development of the nervous system might also, if defective, contribute to infertility. With a role identified for LIS1 in mediating pronuclear migration and genomic union, we might now add fertilization defects together with lissencephaly as devastating consequences induced, respectively, by faulty LIS1 protein and gene expression. 


\section{ACKNOWLEDGMENTS}

We thank Laura Hewitson, Chris Navara, Vanesa Rawe, and Cal Simerly for experimental assistance and helpful discussions. J.R.-S. is supported by a grant from FCT, Portugal (POCTI/ESP/38049/2001). This work was supported by NIH research grants to G.S.

\section{REFERENCES}

Cahana A, Reiner O. 1999. LIS1 and platelet-activating factor acetylhydrolase (Ib) catalytic subunits, expression in the mouse oocyte and zygote. FEBS Lett 451:99-102.

Coquelle FM, Caspi M, Cordelieres FP, Dompierre JP, Dujardin DL, Koifman C, Martin P, Hoogenraad CC, Akhmanova A, Galjart N, De Mey JR, Reiner, O. 2002. LIS1, CLIP-170's key to the dynein/dynactin pathway. Mol Cell Biol 22:3089-3102.

Dawe AL, Caldwell KA, Harris PM, Morris NR, Caldwell GA. 2001. Evolutionarily conserved nuclear migration genes required for early embryonic development in Caenorhabditis elegans. Dev Genes Evol 211:434-441.

Dean B, Chang S, Stevens J, Thomas PE, King C. 2002. Isolation and characterization of a UDP-glucuronosyltransferase (UGT1A01) cloned from female rhesus monkey. Arch Biochem Biophys 402:289-295.

Dobyns WB, Reiner O, Carrozzo R, Ledbetter DH. 1993. Lissencephaly: a human brain malformation associated with deletion of the LIS1 gene located at chromosome 17p13. JAMA 270:2838-2842.

Faulkner NE, Dujardin DL, Tai CY, Vaughan KT, O'Connell CB, Wang Y, Vallee RB. 2000. A role for the lissencephaly gene LIS1 in mitosis and cytoplasmic dynein function. Nat Cell Biol 2:784-791.

Geiser JR, Schott EJ, Kingsbury TJ, Cole NB, Totis LJ, Bhattacharyya G, He L, Hoyt MA. 1997. Saccharomyces cerevisiae genes required in the absence of the CIN8-encoded spindle motor act in functionally diverse mitotic pathways. Mol Biol Cell 8:1035-1050.

Harlow E, Lane D. 1988. Antibodies: a laboratory manual. Cold Spring Harbor, NY: Cold Spring Harbor Laboratory Press.

Hewitson L, Takahashi D, Dominko T, Simerly C, Schatten G. 1998. Fertilization and embryo development to blastocysts by intracytoplasmic sperm injection in the rhesus monkey. Hum Reprod 13:2786-2790.

Hirotsune S, Fleck MW, Gambello MJ, Bix GJ, Chen A, Clark GD, Ledbetter DH, McBain CJ, Wynshaw-Boris A. 1998. Graded reduction of Pafah1b1 (Lis1) activity results in neuronal migration defects and early embryonic lethality. Nat Genet 19:333-339.

Lo Nigro C, Chong CS, Smith AC, Dobyns WB, Carrozzo R, Ledbetter DH. 1997. Point mutations and an intragenic deletion in LIS1, the lissencephaly causative gene in isolated lissencephaly sequence and Miller-Dieker syndrome. Hum Mol Genet 6:157-164.

Memili E, First NL. 1999. Control of gene expression at the onset of bovine embryonic development. Biol Reprod 61:1198-1207.

Morris MC, Depollier J, Mery J, Heitz F, Divita G. 2001. A peptide carrier for the delivery of biologically active proteins into mammalian cells. Nat Biotech 19:1173-1176.

Navara CS, First NL, Schatten G. 1994. Microtubule organization in the cow during fertilization, polyspermy, parthenogenesis, and nuclear transfer: the role of the sperm aster. Dev Biol 162:29-40.

Payne C, Schatten G. 2003. Golgi dynamics during meiosis are distinct from mitosis and are coupled to endoplasmic reticulum dynamics until fertilization. Dev Biol 26: (in press).

Payne C, Rawe V, Ramalho-Santos J, Simerly C, Schatten G. 2003. Preferentially localized dynein and perinuclear dynactin associate with nuclear pore complex proteins to mediate genomic union during mammalian fertilization. J Cell Sci 116: (in press).

Pires-daSilva A, Nayernia K, Engel W, Torres M, Stoykova A, Chowdhury K, Gruss P. 2001. Mice deficient for spermatid perinuclear RNA-binding protein show neurologic, spermatogenic, and sperm morphological abnormalities. Dev Biol 233:319-328.

Reinsch S, Karsenti E. 1997. Movement of nuclei along microtubules in Xenopus egg extracts. Curr Biol 7:211-214.

Richter JD. 2001. Think globally, translate locally: what mitotic spindles and neuronal synapses have in common. Proc Natl Acad Sci USA 98:7069-7071.

Salina D, Bodoor K, Eckley DM, Schroer TA, Rattner JB, Burke B. 2002. Cytoplasmic dynein as a facilitator of nuclear envelope breakdown. Cell 108:97-107.

Sapir T, Elbaum M, Reiner O. 1997. Reduction of microtubule catastrophe events by LIS1, platelet-activating factor acetylhydrolase subunit. EMBO J 16:6977-6984.

Schatten G. 1994. The centrosome and its mode of inheritance: the reduction of the centrosome during gametogenesis and its restoration during fertilization. Dev Biol 165:299-335.

Smith DS, Niethammer M, Ayala R, Zhou Y, Gambello MJ, Wynshaw-Boris A, Tsai LH. 2000. Regulation of cytoplasmic dynein behaviour and microtubule organization by mammalian Lis1. Nat Cell Biol 2:767-775.

Swan A, Nguyen T, Suter B. 1999. Drosophila Lissencephaly-1 functions with Bic-D and dynein in oocyte determination and nuclear positioning. Nat Cell Biol 1:444-449.

Tai CY, Dujardin DL, Faulkner NE, Vallee RB. 2002. Role of dynein, dynactin, and CLIP-170 interactions in LIS1 kinetochore function. J Cell Biol 156:959-968.

Xiang X, Morris NR. 1999. Hyphal tip growth and nuclear migration. Curr Opin Microbiol 2:636-640.

Xiang X, Osmani AH, Osmani SA, Xin M, Morris NR. 1995. NudF, a nuclear migration gene in Aspergillus nidulans, is similar to the human LIS-1 gene required for neuronal migration. Mol Biol Cell 6:297-310. 